\title{
A CLASS OF TWO GENERATOR TWO RELATION FINITE GROUPS
}

J. W. WAMSLEY

(Received 13 July 1970)

Communicated by G. E. Wall

\section{Introduction}

A group which is minimally generated by $n$ generators and defined by $n$ relations is said to have zero deficiency. The class of finite groups known to have zero deficiency is small, consisting of cyclic groups, certain metacyclic groups [4] and classes of groups given in [1], [2] and [3].

In this paper we give a class of 2 generator 2 relation finite groups which does not appear to be contained in the known classes. The class of groups considered is denoted $\{G(\alpha, \beta, \gamma)\}$, with presentation

$$
G(\alpha, \beta, \gamma)=\left\{a, b \mid b a b^{-1}=a^{\alpha} b^{\beta}, a^{-1} b^{\beta} a=b^{\gamma \beta}\right\},
$$

which is shown to be finite for $\alpha, \gamma>1$. Also we show that each element of $G(\alpha, \beta, \gamma)$ may be written as $a^{r} b^{s}$ for suitable $r$ and $s$ but that $G(\alpha, \beta, \gamma)$ is in general not metacyclic.

\section{Finiteness of $G(\alpha, \beta, \gamma)$}

The relations are

$$
\begin{aligned}
& b a b^{-1}=a^{\alpha} b^{\beta}, \quad \alpha>1 \quad \text { and } \\
& a^{-1} b^{\beta} a=b^{\gamma \beta}, \quad \gamma>1 .
\end{aligned}
$$

From (2) we have immediately

$$
\begin{gathered}
a^{-r} b^{s \beta} a^{r}=b^{t}, \quad t=\mathrm{s} \beta \gamma^{r} \text { for } r>0 \text { giving } \\
b^{\beta+1} a=b\left(b^{\beta} a\right)=(b a) b^{\gamma \beta}=a^{\alpha} b^{1+\beta+\gamma \beta} \\
=b^{\beta}(b a)=\left(b^{\beta} a^{\alpha}\right) b^{1+\beta}=a^{\alpha} b^{1+\beta+\beta \gamma^{\alpha}} \quad \text { whence } \\
b^{\gamma \beta\left(\gamma^{\alpha-1}-1\right)}=1,
\end{gathered}
$$

and since $a b^{\gamma \beta} a^{-1}=b^{\beta}$ then 


$$
b^{\beta\left(\gamma^{\alpha-1}-1\right)}=1 \text {. }
$$

Consider the normal subgroup $B=\left\langle b^{\beta}\right\rangle$ of $G(\alpha, \beta, \gamma)$. Let $G_{1}=G / B, a_{1}=a B$, $b_{1}=b B$. Then $G_{1}$ has presentation

$$
\left\{a_{1}, b_{1} \mid b_{1} a_{1} b_{1}^{-1}=a_{1}^{\alpha}, b_{1}^{\beta}=1\right\}
$$

and is therefore metacyclic of order $\beta\left(\alpha^{\beta}-1\right)$.

Since each element of $G_{1}$ has the form

$$
a_{1}^{r_{1}} b_{1}^{s_{1}}\left(0 \leqq r_{1}<\alpha^{\beta}-1,0 \leqq s_{1}<\beta\right),
$$

each element of $G$ has the form

$$
a^{r} b^{s}\left(0 \leqq r<\alpha^{\beta}-1,0 \leqq s<\beta\left(\gamma^{\alpha-1}-1\right)\right) .
$$

Hence $G$ is finite of order $\leqq\left(\alpha^{\beta}-1\right) \beta\left(\gamma^{\alpha-1}-1\right)$.

\section{An example of $G(\alpha, \beta, \gamma)$}

To show that $G(\alpha, \beta, \gamma)$ is in general not metacyclic we look more closely at

$$
G(m, m-1, m) \text {. }
$$

Consider the set, $G$, of ordered pairs $(\alpha, \beta)$ with $0 \leqq \alpha, \beta<n$ where $n=m^{m-1}-1$.

Define multiplication by

$$
\begin{aligned}
& (\alpha, \beta)(\gamma, \delta)=(x, y) \text { where } \\
& x \equiv \alpha+\gamma m^{\beta} \text { modulo } n \text { and } \\
& y \equiv \delta+\beta m^{\gamma} \text { modulo } n .
\end{aligned}
$$

Then $G$ becomes a group generated by $(1,0)$ and $(0,1)$ with

$$
\begin{aligned}
(0,1)(1,0)(0,1)^{-1} & =(1,0)^{m}(0,1)^{m-1} \text { and } \\
(1,0)^{-1}(0,1)^{m-1}(1,0) & =(0,1)^{m(m-1)} \text { whence }
\end{aligned}
$$

$G$ is a factor group of $G(m, m-1, m)$.

Let $a=(0,1), b=(1,0)$ then the elements

lie in $G^{\prime}$. Since

$$
\begin{aligned}
& a^{-1} b a b^{-1}=a^{\alpha-1} b^{\beta}=(m-1, m-1) \text { and } \\
& b^{-\beta} a^{-1} b^{\beta} a=b^{(\gamma-1) \beta}=\left(0,(m-1)^{2}\right)
\end{aligned}
$$

$$
\begin{aligned}
(m-1, m-1)^{t} & =(t(m-1), t(m-1)) \text { and } \\
\left(0,(m-1)^{2}\right)^{t} & =\left(0, t(m-1)^{2}\right),
\end{aligned}
$$

these elements generate trivially interesting cyclic subgroups of orders $n /(m-1)$ and $n /(m-1)^{2}$. Hence $G^{\prime}$ is not cyclic and so $G(m, m-1, m)$ cannot be metacyclic.

I should like to extend sincere thanks to the referee for his comments. 


\section{References}

[1] I. D. MacDonald, 'On a class of finitely presented groups', Canad. J. Math. 14 (1962), 602-613.

[2] J. Mennicke, 'Einige endlicke Gruppen mit drei Erzeugenden und drei Relationen', Archiv der Math. 10 (1959), 409-418.

[3] J. W. Wamsley, 'A class of three generator three relation finite groups', Canad. J. Math. 22 (1970), 36-40.

[4] J. W. Wamsley, 'The deficiency of metacyclc groups', Proc. Amer. Math. Soc. 24 (1970), 724-726.

School of Mathematical Sciences

The Flinders University of South Australia

Beford Park, South Australia, 5042, Australia 